\title{
Some Observations on Damage Tolerance Analyses in Pressure Vessels
}

\author{
Ivatury S. Raju ${ }^{1}$ \\ NASA Langley Research Center, Hampton, Virginia, 23681 \\ David S. Dawicke ${ }^{2}$ \\ AS\&M, Inc., Hampton, Virginia, 23666 \\ and \\ Roy W. Hampton ${ }^{3}$ \\ Owl Analytics, LLC, Cupertino, California, 95014
}

\begin{abstract}
AIAA standards S080 and S081 are applicable for certification of metallic pressure vessels (PV) and composite overwrap pressure vessels (COPV), respectively. These standards require damage tolerance analyses with a minimum reliable detectible flaw/crack and demonstration of safe life four times the service life with these cracks at the worst-case location in the PVs and oriented perpendicular to the maximum principal tensile stress. The standards require consideration of semi-elliptical surface cracks in the range of aspect ratios (crack depth $a$ to half of the surface length $c$, i.e., $(a / c)$ of 0.2 to 1$)$. NASA-STD-5009 provides the minimum reliably detectible standard crack sizes $(90 / 95$ probability of detection (POD) for several non-destructive evaluation (NDE) methods (eddy current (ET), penetrant (PT), radiography (RT) and ultrasonic (UT)) for the two limits of the aspect ratio range required by the AIAA standards. This paper tries to answer the questions: can the safe life analysis consider only the life for the crack sizes at the two required limits, or endpoints, of the $(a / c)$ range for the NDE method used or does the analysis need to consider values within that range? What would be an appropriate method to interpolate 90/95 POD crack sizes at intermediate $(a / c)$ values? Several procedures to develop combinations of $a$ and $c$ within the specified range are explored. A simple linear relationship between $a$ and $c$ is chosen to compare the effects of seven different approaches to determine combinations of $a_{j}$ and $c_{j}$ that are between the $(a / c)$ endpoints. Two of the seven are selected for evaluation: Approach $I$, the simple linear relationship, and a more conservative option, Approach III. For each of these two Approaches, the lives are computed for initial semi-elliptic crack configurations in a plate subjected to remote tensile fatigue loading with an R-ratio of 0.1 , for an assumed material evaluated using NASGRO ${ }^{\circledR 4}$ version 8.1. These calculations demonstrate that for this loading, using Approach I and the initial detectable crack sizes at the $(a / c)$ endpoints in 5009 specified for the ET and UT NDE methods, the smallest life is not at the two required limits of the $(a / c)$ range, but rather is at an intermediate configuration in the range $(a / c)$ of 0.4 to 0.6. Similar analyses using both Approach I and III with the initial detectable crack size at the $(a / c)$ endpoints in $\mathbf{5 0 0 9}$ for PT NDE showed the smallest life may be at an $(a / c)$ endpoint or an intermediate $(a / c)$, depending upon which Approach is used. As such, analyses that interrogate only the two $(a / c)$ values of 0.2 and 1 may result in unconservative life predictions. The standard practice may need to be revised based on these results.
\end{abstract}

\footnotetext{
${ }^{1}$ NASA Technical Fellow for Structures, NASA Engineering and Safety Center. Fellow AIAA, Member ASME, Member ASCE.

${ }^{2}$ Senior Scientist

${ }^{3}$ Consultant, Member ASTM/ASME.

${ }^{4}$ NASGRO $^{\circledR} 8.1$ is a Fracture Mechanics and Fatigue Crack Growth Analysis Software developed and distributed under the terms of a Space Act Agreement between NASA Johnson Space Center and Southwest Research Institute.
}

1

American Institute of Aeronautics and Astronautics 


\section{Introduction}

【 IGHT weight metallic pressure vessels (PV) and Composite Overwrap Pressure Vessels (COPVs) are used in

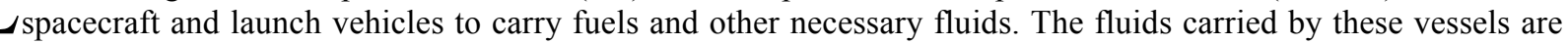
under high pressure and failure of a PV or a COPV will likely lead to loss of mission for robotic missions and loss of mission and life for human rated missions. Hence, the structural integrity of the PVs and COPVs is important. During the past three decades, considerable progress has been made in designing, operating, and predicting failures of these PVs ${ }^{1-3}$.

In this paper, some observations on damage tolerance (DT) of PVs and COPVs are presented. Linear elastic fracture mechanics (LEFM) is used to evaluate life and residual strength of the metallic liner. As is customary in fracture mechanics, stress analyses are performed for the maximum design pressure (MDP) in the vessel. Regions of high stresses and large stress gradients are identified. In the region of the highest stresses, semi-elliptic surface (part through) cracks are introduced and oriented perpendicular to the maximum normal tensile principal stress. The growth of these cracks is then evaluated for spectrum loading that the PV experiences in its lifetime. While the processes that need to be followed are clear from the AIAA standards $\mathrm{S} 080^{2}$ for metallic PVs, and $\mathrm{S} 081^{3}$ for COPVs, implementation details are often fuzzy and are open for interpretation by the users/analysts. This paper examines a few areas where implementation issues arise and offers some guidance with regard to LEFM calculations, assumed initial detectible crack sizes, fatigue and fracture considerations (i.e., safe-life) and the use of, and limitations of, LEFM.

\section{Pressure Vessel Configurations}

Figure 1 shows a cylindrical metallic PV and two COPVs. COPVs have a metallic liner and a composite overwrap that is wound over the liner. Figure 2 shows idealized cross section views of a COPV. The pressure vessels can be formed by welding parts of metallic segments together as shown in Fig. 3, or other techniques (e.g., spin forming). As mentioned previously the PVs need to comply with standards and requirements laid out in AIAA S080 and AIAA S081A standards.

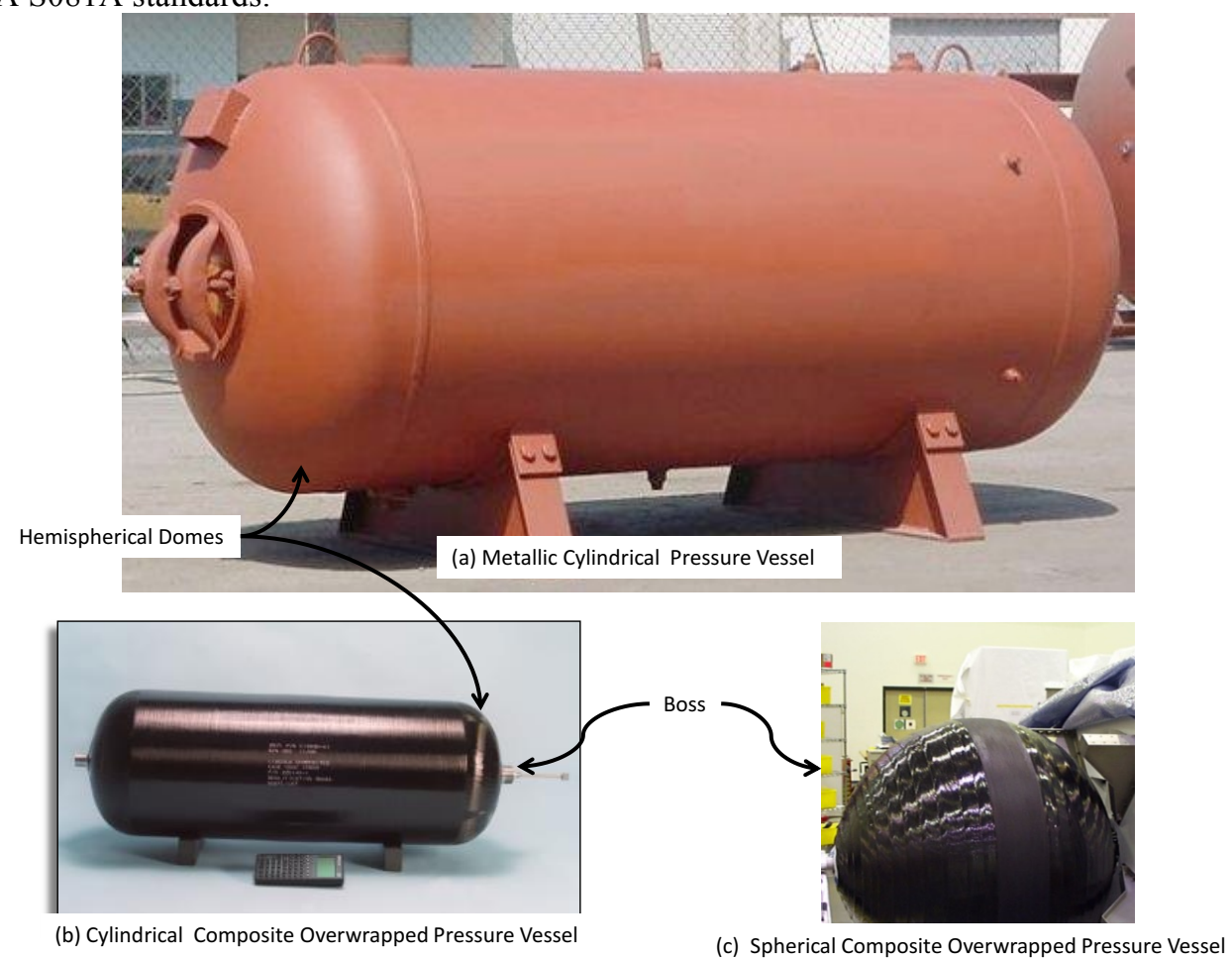

Figure 1. Example of metallic pressure vessels (PV) and composite overwrapped pressure vessels (COPV). 


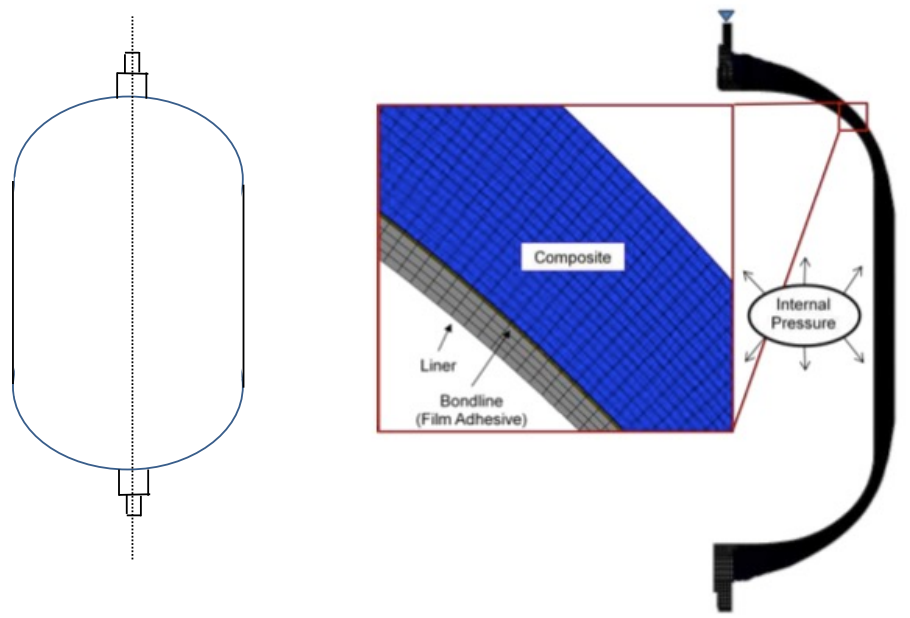

Figure 2. A COPV and its cross section.

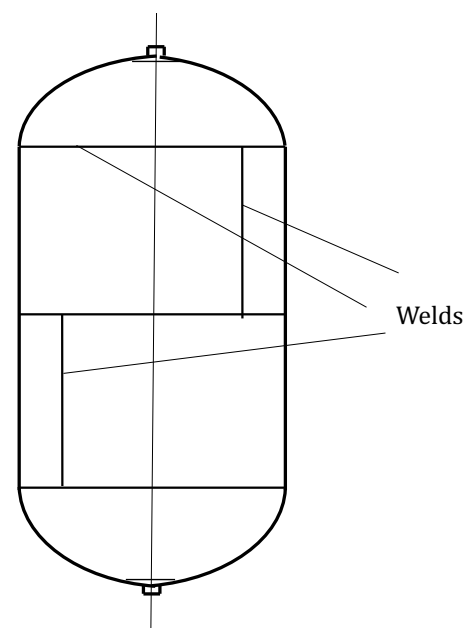

Figure 3. Welded pressure vessel.

\section{Damage Tolerance Requirements}

The damage tolerance requirements in the standards specify that all areas of the metallic liner except the shear region of the boss shall possess a minimum damage tolerance life (safe-life) of 4 lifetimes under the spectrum loading the PV experiences during its lifetime. The standard also requires demonstration of residual strength at MDP. That is, the maximum stress intensity factor at any point on the crack front of the semi-elliptic crack must be less than the condition resulting in fracture of the material, that may be the fracture toughness of the material, $\mathrm{K}_{\mathrm{IC}}$, or other relevant parameters listed in the later section titled "Other Considerations." The assumed initial flaw (crack) size and shape for both safe-life and residual strength is based on nondestructive evaluation capability (i.e., a crack size with an estimated probability of detection of $90 \%$ with $95 \%$ confidence). The NASA-STD-5009 Table $1^{4}$ shows the NDE capabilities and the corresponding size of the cracks that can be reliably detected with "Standard" NDE methods and qualified inspectors. A version of this table is shown in Table 1. The data is derived from historical studies that estimated the $90 \%$ probability of detection at a $95 \%$ confidence level for detecting cracks in flat plates by the NDE methods as described in the standard. A crack that is the size estimated by the 90/95 POD for the given NDE inspection is presumed to exist in the structure. Different NDE techniques may be used at different locations on the vessels. Thus, the size and shape of the initial flaw that is used to demonstrate safe-life can be different at different locations. In addition, the safe-life demonstration must consider how the thickness and the material properties vary at different locations (e.g., acreage, attachment bosses, welds). Also, localized stresses may include significant bending as well as tension stresses in regions of geometric shape changes or curvature, and in addition, welding residual stresses may be present at welded joints. All these variables need to be accounted for in the DT analyses. For the analyses reported here, the effects of a set of simple remote tension and bending loading examples are evaluated and discussed.

Table 1. NDE Methods and detectability limits.

\begin{tabular}{|c|c|c|c|c|}
\hline Crack Location & Part Thickness, t, in. & Crack type & Crack Dimension, a, in. & Crack Dimension, c, in. \\
\hline \multicolumn{5}{|c|}{ Eddy Current } \\
\hline \multirow[t]{3}{*}{ Open Surface } & $\mathrm{t}<=0.050$ & Through & $\mathrm{t}$ & 0.050 \\
\hline & $t>0.050$ & PTC & 0.020 & 0.100 \\
\hline & & & 0.050 & 0.050 \\
\hline \multicolumn{5}{|c|}{ Penetrant NDE } \\
\hline \multirow[t]{4}{*}{ Open Surface } & $t<=0.050$ & Through & $\mathrm{t}$ & 0.100 \\
\hline & $0.050<\mathrm{t}<0.0750$ & Through & $\mathrm{t}$ & $0.150-\mathrm{t}$ \\
\hline & $T>0.0750$ & PTC & 0.025 & 0.125 \\
\hline & & & 0.075 & 0.075 \\
\hline \multicolumn{5}{|c|}{ Radiographic NDE } \\
\hline \multirow[t]{3}{*}{ Open Surface } & $\mathrm{t}<=0.107$ & PTC & $0.7 \mathrm{t}$ & 0.075 \\
\hline & $T>0.107$ & PTC & $0.7 \mathrm{t}$ & $0.7 \mathrm{t}$ \\
\hline & & Embedded & $2 a=.7 t$ & $0.7 \mathrm{t}$ \\
\hline \multicolumn{5}{|c|}{ Ultrasonic } \\
\hline \multirow[t]{4}{*}{ Open Surface } & $t>=0.100$ & PTC & 0.030 & 0.150 \\
\hline & & & 0.065 & 0.065 \\
\hline & & Embedded & 0.017 & 0.087 \\
\hline & & & 0.039 & 0.039 \\
\hline
\end{tabular}

3

American Institute of Aeronautics and Astronautics 


\section{Damage-Tolerance Life (Safe-Life) Analysis}

Linear elastic fracture mechanics (LEFM) is generally used to demonstrate damage tolerance life for metallic PV and COPVs with elastically responding liners. A part-through crack is assumed to be in the most critical locations and is oriented perpendicular to the highest principal stress in the vessel. The flaw (crack) shape $(a / c)$ in the range of 0.2 to 1.0 are considered (see Fig. 4).

A state-of-the-art crack growth software package such as NASGRO ${ }^{\circledR 5}$ is generally used to conduct the analysis. As the crack grows under cyclic loading, the flaw shape changes are accounted for in the analyses. Details used in the LEFM analyses such as plane strain fracture toughness $\left(\mathrm{K}_{\mathrm{Ic}}\right)$ and fatigue crack growth rates, $(d a / d N)$; the loading spectrum and environments; NDE methods and corresponding initial flaw sizes; and a summary of significant

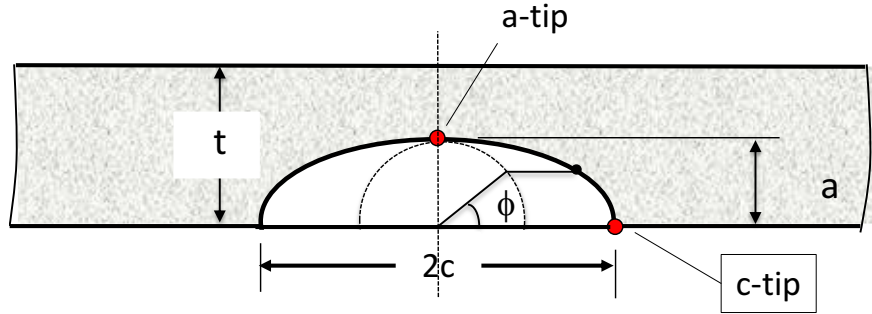

Figure 4. Semi-elliptic surface crack.

results are reported in the DT analyses. Both AIAA S080 and S081 standards also allows for the demonstration of DT life through testing of coupons or flight representative COPVs.

\section{Assumptions}

Damage tolerance safe-life analyses, as is practiced today, has the fundamental assumption that LEFM is valid. The underlying LEFM assumptions are:

1. The material of the vessel away from the crack tip is undergoing elastic deformations.

2. The plastic region ahead of the crack tip/front is small in relation to the length of the crack.

3. Deformations outside the small plastic regions such as the remaining ligament between the crack front and the far surface of the thickness are presumed to remain elastic.

4. The material is presumed to have a grain structure such that the integrated effects of the grains in these regions acts like a continuum.

These LEFM assumptions are generally valid when the crack depth is small compared to the thickness. However, the limitations of these assumptions (i.e., what is considered small for the ratio of crack depth to thickness) have not been established by the standards. Thus, the user is responsible to verify that LEFM assumptions are appropriate for the analysis being conducted. The violation of the LEFM assumptions, as will eventually occur as the liner thickness is reduced, may lead to unconservative results. This paper presumes conditions of LEFM applicability.

\section{Critical Question}

While the standard appears to be clear on the procedures/processes needed for demonstrating safe-life, various questions arise when implementing these requirements. For example, how is the requirement to examine cracks 'in the range of $(a / c)=1.0$ and $0.2^{\prime}$ interpreted? Does the stress-intensity factor need to be examined at every point on the crack front? Where is the stress-intensity factor maximum along the crack front? Are the conclusions drawn for a particular liner region thickness valid for the entire liner? If not, what range are these conclusions valid? The remainder of the paper attempts to answer some of these questions.

Maximum K locations: Where along the crack front of a part through crack (PTC), also called a surface crack, is the stress-intensity factor maximum? The maximum stress-intensity factor $(\mathrm{K})$ location varies with aspect ratios ( $\operatorname{such}$ as $(a / c)=0.2,0.4,0.6,0.8,1.0)$ and with crack depths (such as $(a / t)=0.2,0.4,0.6,0.8)^{6-8}$. The maximum $\mathrm{K}$ location also depends upon the applied loading (tension and bending, etc.). From previous work in references 6-8, this maximum $\mathrm{K}$ location was identified to occur only at either the $c$-tip or the $a$-tip for the tension loading condition. Figure 5 (a) - (d) presents the normalized values of $\mathrm{K}$ for remote tensile loading, $\mathrm{S}_{\mathrm{t}}$, in a flat plate for various crack depths $(a / t) ;(a / t)=0.2$ represents a shallow crack while $(a / t)=0.8$ represents a deep crack. In these figures the value of $\mathrm{K}$ at the $c$-tip $(\phi=0)$ and a-tip $(\phi=\pi / 2)$ are plotted. Figure 5 demonstrates that for $(a / c)=0.2$ and 0.4 the maximum value of $\mathrm{K}$ occurs at the deepest point, $a$-tip $(\phi=\pi / 2)$ for all crack depths. In contrast, for $(a / c)=1$ the maximum value always occurs at the $c$-tip, $(\phi=0)$. For the crack shape corresponding to $(a / c)=0.6$, however, the behavior is mixed. For $(a / t)=0.2$ through 0.6 , the maximum value of $\mathrm{K}$ occurs at the deepest point $(\phi=\pi / 2)$, while for $(a / t)=0.8$ the maximum value occurs at the $c$-tip, $(\phi=0)$. This also demonstrates that the maximum values occur at either the $a$-tip or the $c$-tip and not elsewhere on the crack front. Thus, the DT life must only be interrogated at the $a$-tip and the $c$-tip. 


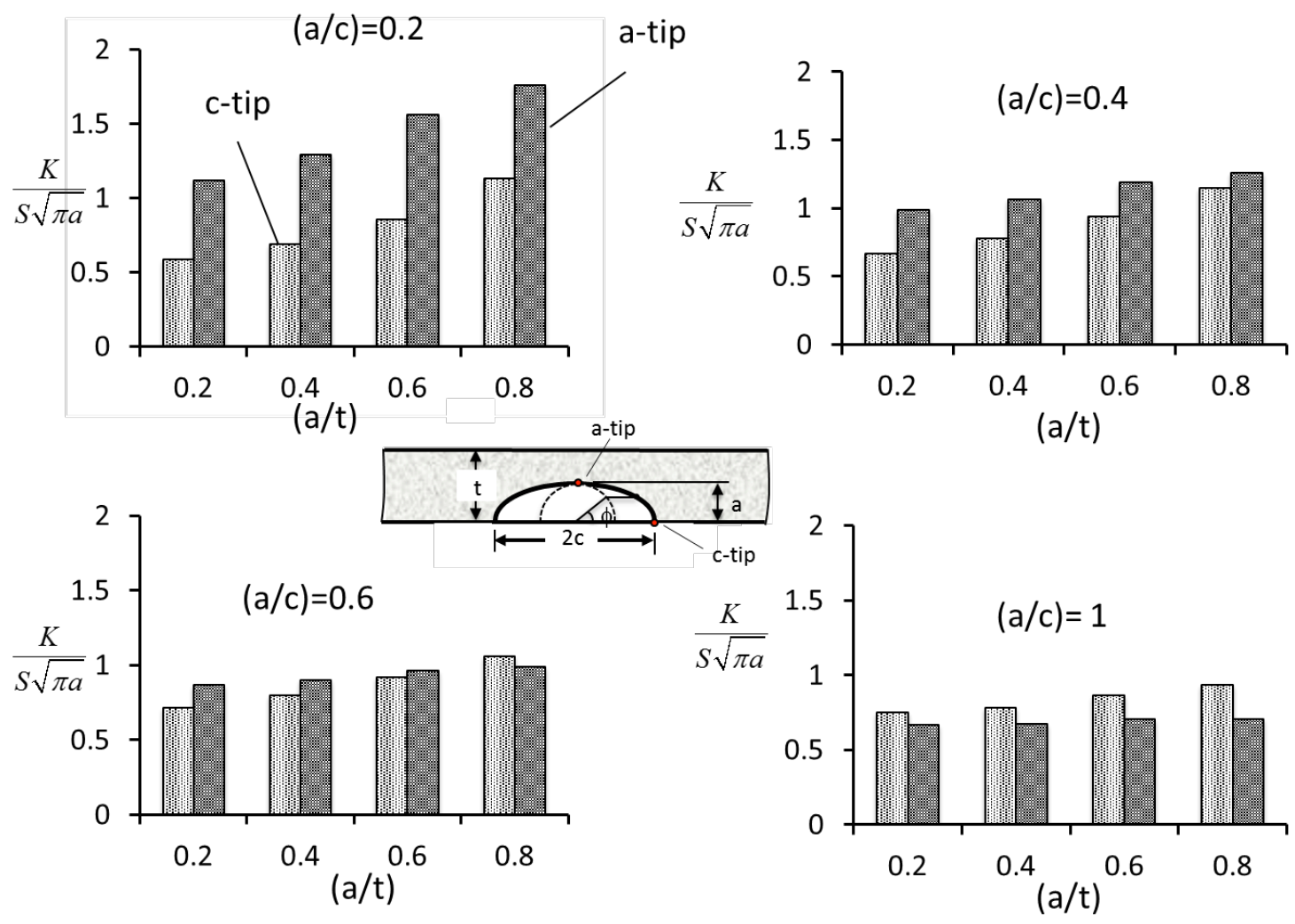

Figure 5. Normalized Stress-intensity factors for various crack shapes at c- and a-tips for remote tensile loading, $\mathbf{S}$.

Loadings: For other loading cases, the behavior depends on the combined loadings. Examples reported here include a pure tension, $\mathrm{S}_{\mathrm{t}}$, and several combinations of tension $\mathrm{S}_{\mathrm{t}}$ plus pure bending, $\mathrm{S}_{\mathrm{b}}$. Figure 6 illustrates these loadings that cause crack opening at the $c$-tip, and more complex conditions at the $a$-tip. In practice, either $\mathrm{S}_{\mathrm{t}}$ or $\mathrm{S}_{\mathrm{b}}$ or both may be reversed, causing compression on the crack $c$-tip. More complex stress fields also may be encountered, such as those due to residual stress effects. For these cases, computer analyses must be used that compute the K values due to the integrated effect of local stresses acting at the $c$-tip and $a$-tip. These $\mathrm{K}$ values are then used to predict crack growth rates at each crack tip, the incremental crack growth at each tip, and the resulting progressive change of crack shape during fatigue crack growth.

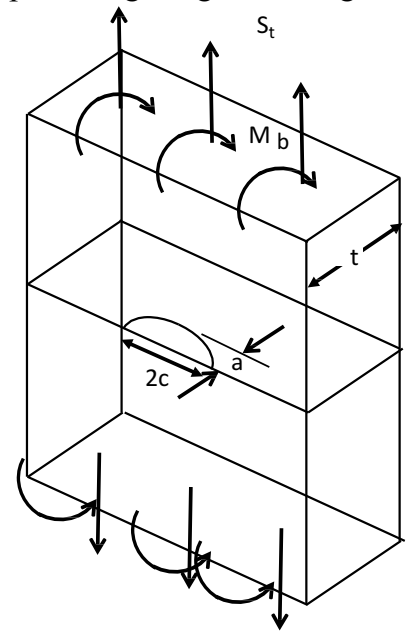

(a) Surface crack in a plate subjected to remote tension, $\mathrm{S}_{\mathrm{t}}$ and bending $\mathrm{M}_{\mathrm{b}}$.

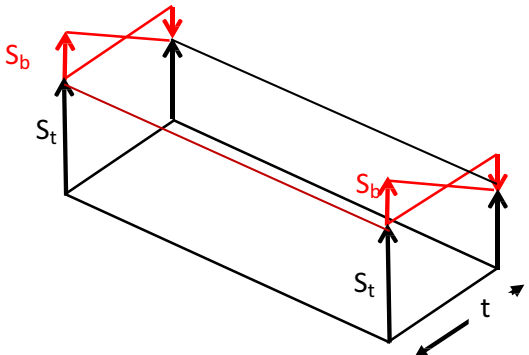

(b) Remote tension $\mathrm{S}_{\mathrm{t}}$ and bending $\mathrm{S}_{\mathrm{b}}$ loading on the top and bottom faces of the plate.

Figure 6. Surface crack in a plate with tension, $S_{t}$, and bending loadings $M_{b}$. 


\section{Crack Shapes and Sizes}

NASA-Standard- $5009^{4}$ discusses NDE requirements for fracture critical components. All pressure vessels and COPVs are classified as fracture critical ${ }^{9}$. The 5009 NDE standard lists several NDE methodologies and the limitations of their ability to detect part through cracks (surface) and embedded cracks. The NDE methodologies addressed here are eddy current (ET), penetrant (PT), radiography (RT), and ultrasonic (UT). Table 1 summarizes the $5009 \mathrm{NDE}$ standard for each of these NDE techniques and the associated minimum detectible flaw sizes (based on an estimated 90/95 POD) for through the thickness cracks, surface cracks, and embedded cracks. Each of the NDE techniques has a different minimum detectible flaw size and each of these techniques is applicable to particular thicknesses of metallic PVs and COPV liners. Surface cracks could be on the internal or the external metallic surfaces. Embedded cracks at joints could be centrally located through the thickness of the metal, or could be offset from the center. The DT analyses need to account for all these cases.

Table 1 provides the minimum detectible flaw sizes at the extreme aspect ratios, or endpoints in this analysis for "Standard NDE" per $5009^{5}$. The safe-life requirement is that the most damaging crack configuration be considered. None of the standards describe how to interpolate between the NDE detectable crack aspect ratio endpoints, $(a / c)=0.2$ and 1 , to determine the most damaging crack configuration. Further, there is little or no known POD data for the various NDE methods at intermediate $(a / c)$ ratio values that can be used to base any interpolation between the endpoints. As such, seven approaches to determine initial crack sizes between the $(a / c)$ endpoints are studied. These simple approaches are described below as to whether they are conservative relative to each other. Other considerations are discussed later. These approaches are illustrated in Fig. 7 for the penetrant NDE method detectable crack sizes at the crack aspect ratio endpoints in 5009.

Approach I: $a=m c+r$

This approach assumes that the crack depth $(a)$ is a linear function of the crack length $(c)$ between the two $(a / c)$ ratio endpoints, $(a / c)=0.2$ and 1 . The slope $m$ and the intercept $r$ of the straight line are derived from the crack depth and length at the two endpoints. The resulting straight line is more conservative than Approach II but less conservative than Approach III (discussed below). This method was originally proposed in the Space Shuttle Orbiter Fracture Control Plan ${ }^{10}$.

Approach II: $a=m(a / c)+r$

This approach assumes that the crack depth $(a)$ is a linear function of the aspect ratio $(a / c)$ between the two $(a / c)$ ratio endpoints, $(a / c)=0.2$ and 1 . The slope $m$ and the intercept $r$ of the straight line are derived from the crack depth and aspect ratio at the two endpoints. The resulting curve has a non-linear relationship between $(a)$ and $(c)$. This relationship produces smaller interpolated crack sizes and is therefore nonconservative relative to Approach I.

Approach III: $c=m(a / c)+r$

This approach assumes that the crack length $(c)$ is a linear function of the aspect ratio $(a / c)$ between the two a/c endpoints, $(a / c)=0.2$ and 1 . The slope $m$ and the intercept $r$ of the straight line are derived from the crack depth and aspect ratio at the two endpoints. The resulting curve has a non-linear relationship between $(a)$ and $(c)$ and this relationship is conservative relative to Approach I because it produces larger interpolated crack sizes than Approach I.

Approach IV: Area $=m a+r$

This approach assumes that the crack area $(1 / 2 \pi a c)$ is a linear function of the crack depth $(a)$ between the two $(a / c)$ endpoints, $(a / c)=0.2$ and 1 . The slope $m$ and the intercept $r$ of the straight line are derived from calculating the area at each endpoint and fitting a line to the area and crack depth. This approach produces smaller interpolated crack sizes and is less conservative than Approach I.

Approach V: Area $=m c+r$

This approach assumes that the crack area $(1 / 2 \pi a c)$ is a linear function of the surface crack length $(c)$ between the two $(a / c)$ endpoints, $(a / c)=0.2$ and 1 . The slope $m$ and the intercept $r$ of the straight line are derived from

\footnotetext{
${ }^{5}$ Other, smaller crack sizes can be considered if the NDE techniques and operators have passed a POD evaluation to prove capability as "Special NDE" per requirements in section 4.3 in the NASA-STD-5009 4 . It is recognized that most thin wall flight PV are qualified for "Special NDE" to obtain smaller detectability limit crack sizes than are in 5009 Table 1. However, "Special NDE" crack sizes are not published, as they are individually established per 5009 requirements, thus cannot be used for the example analyses reported in this paper. Therefore, the "Standard NDE" crack sizes are used for the assessments reported here. Nevertheless, the analyses reported here are expected to also be applicable for "Special NDE" initial crack sizes, although the results may vary somewhat due to the different initial crack sizes.
} 
calculating the area at each endpoint and fitting a line to the area and surface length. This approach also produces smaller interpolated crack sizes and is less conservative than Approach I.

Approach VI: Area $=$ constant (based on the area for the NDE endpoint at $(a / c)=1$ )

This approach calculates the area at the endpoint at $(a / c)=1$ and assumes that the area is constant for all other aspect ratios. As discussed later in the paper, this approach may be more conservative than Approach I and does not necessarily agree with the value at the $(a / c)=0.2$ endpoint.

Approach VII: Area $=$ constant (based on the area for the NDE endpoint at $(a / c)=0.2$ )

This approach first calculates the area at the endpoint at $(a / c)=0.2$ and assumes that the area is constant for all other aspect ratios. This approach is less conservative than Approach I and does not necessarily agree with the value at the $(a / c)=1$ endpoint.
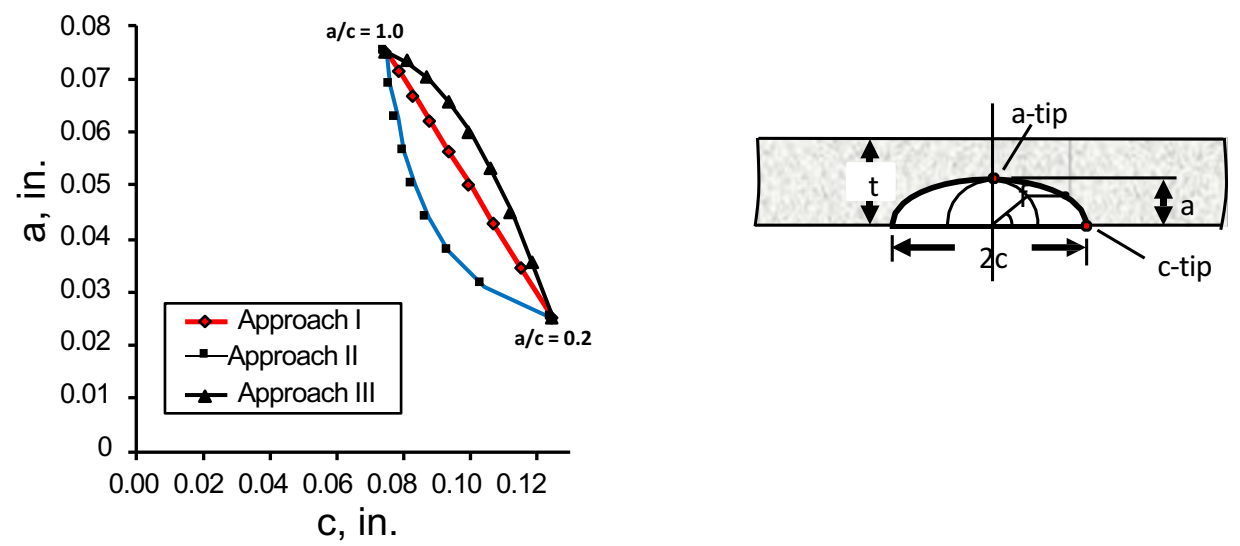

(a) Approaches I, II, and III.

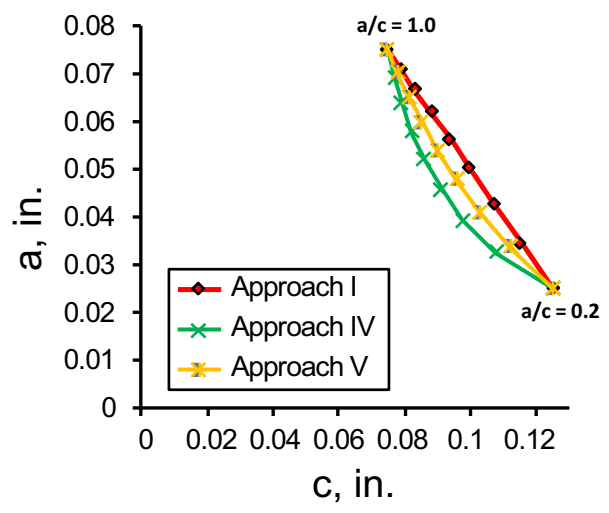

(b) Approaches I, IV, and V.

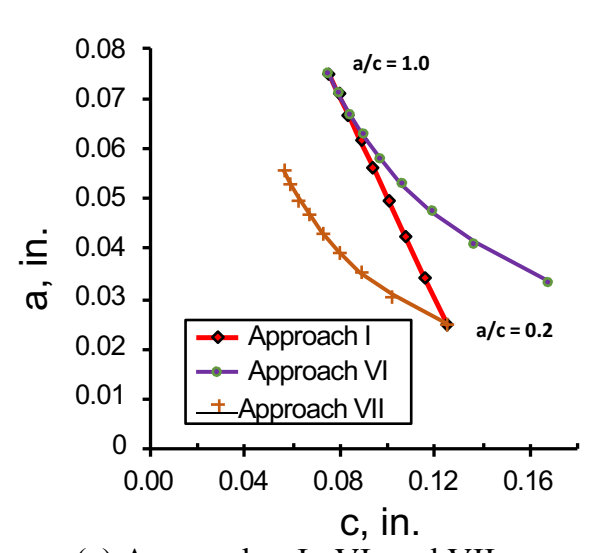

(c) Approaches I, VI, and VII.

Figure 7. Various crack shape interpolation approaches studied using 5009 detectable crack aspect ratio endpoints for penetrant NDE.

\section{Discussion of Various Approaches}

The lack of minimum detectible flaw sizes for the standard NDE methods in 5009 for aspect ratios between the two specified NDE aspect ratio endpoints $(a / c=0.2$ and 1.0) requires an assumption be made when predicting life for cracks between these endpoint sizes. The NDE information is given in terms of crack depth, $a$, and crack halflength, $c$, so the choice was made to assume a linear relationship between these two parameters (Approach I) and perform calculations with results as shown in Figs. 8 through 12, 14, and 15. All other approaches use quantities such as $(a / c)$ or crack area or another assumption. These approaches may be more or less conservative than Approach I as detailed previously and in the following discussion. 

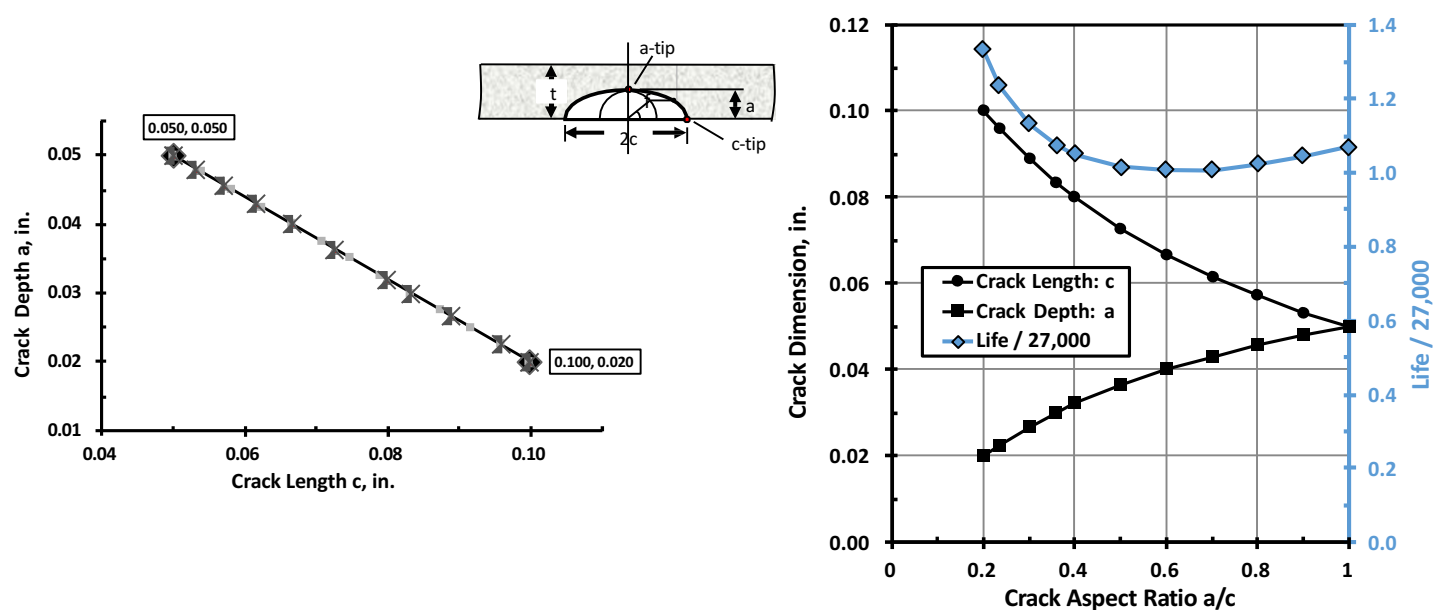

(a) Eddy current NDE aspect ratio endpoints and interpolated shapes using Approach I.

(b) Calculated lives for Approach I interpolated initial crack size and aspect ratios.

Figure 8. Eddy current NDE 5009 detectable crack aspect ratio endpoints, interpolated crack shapes using Approach I, and lives at each aspect ratio (20 ksi remote tension \& plate thickness $=0.075$ in).

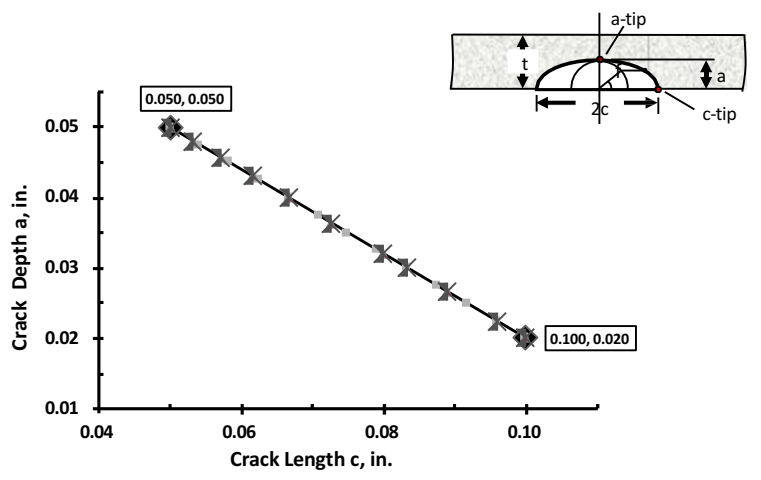

(a) Eddy current NDE aspect ratio endpoints and interpolated shapes using Approach I.

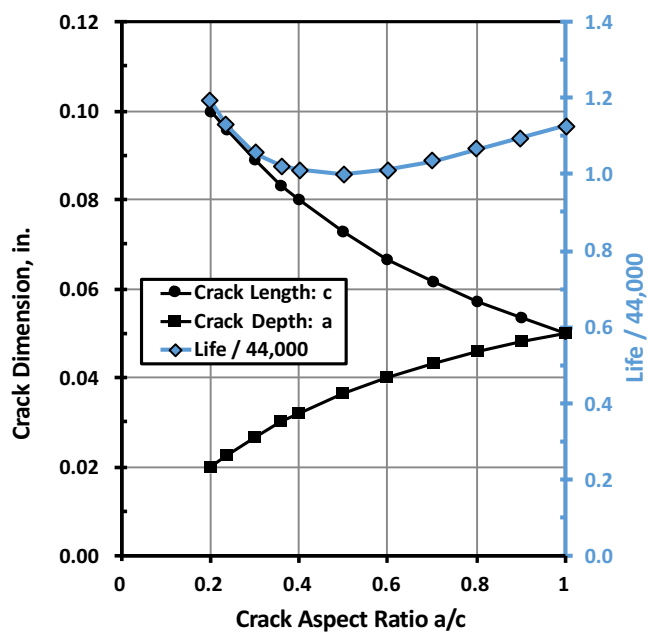

(b) Calculated lives for Approach I interpolated initial crack size and aspect ratios.

Figure 9. Eddy current NDE 5009 detectable crack aspect ratio endpoints, interpolated crack shapes using Approach I, and lives at each aspect ratio (20 ksi remote tension \& plate thickness $=0.1$ in). 

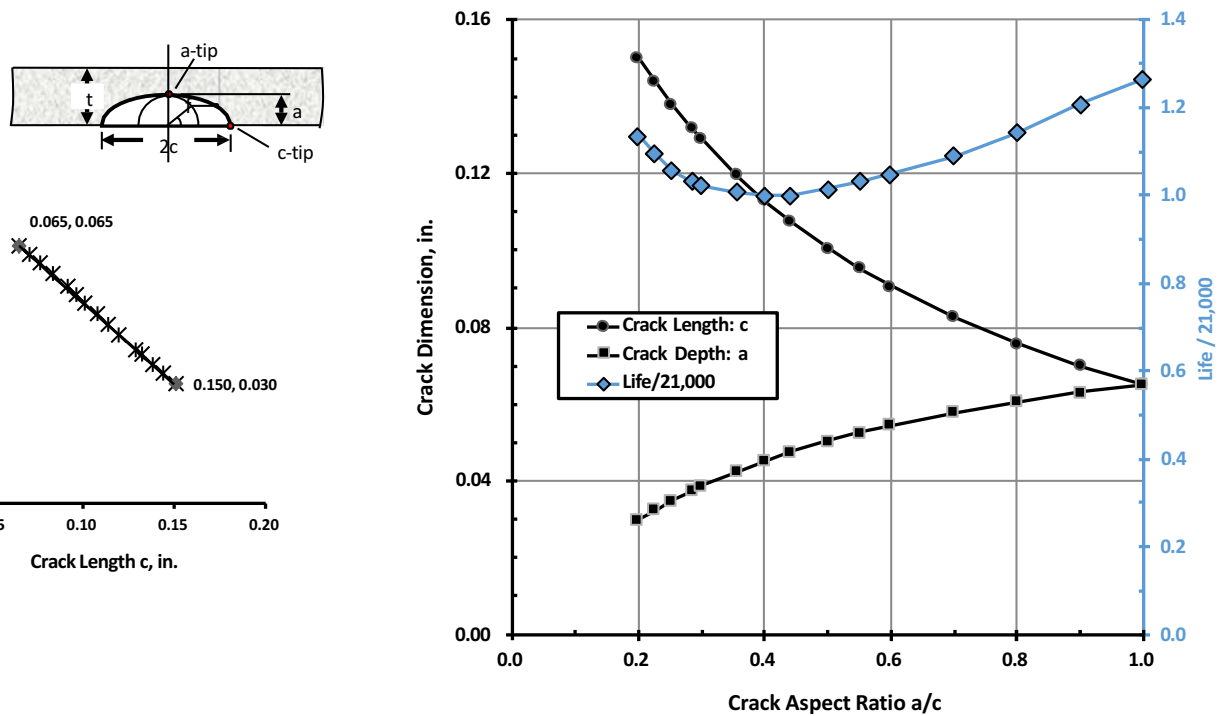

(a) Ultrasonic NDE aspect ratio endpoints and interpolated shapes using Approach I.

(b) Calculated lives for Approach I interpolated initial crack size and aspect ratios.

Figure 10. Ultrasonic NDE 5009 detectable crack aspect ratio endpoints, interpolated crack shapes using Approach I, and lives at each aspect ratio (20 ksi remote tension \& plate thickness $=0.1$ in).
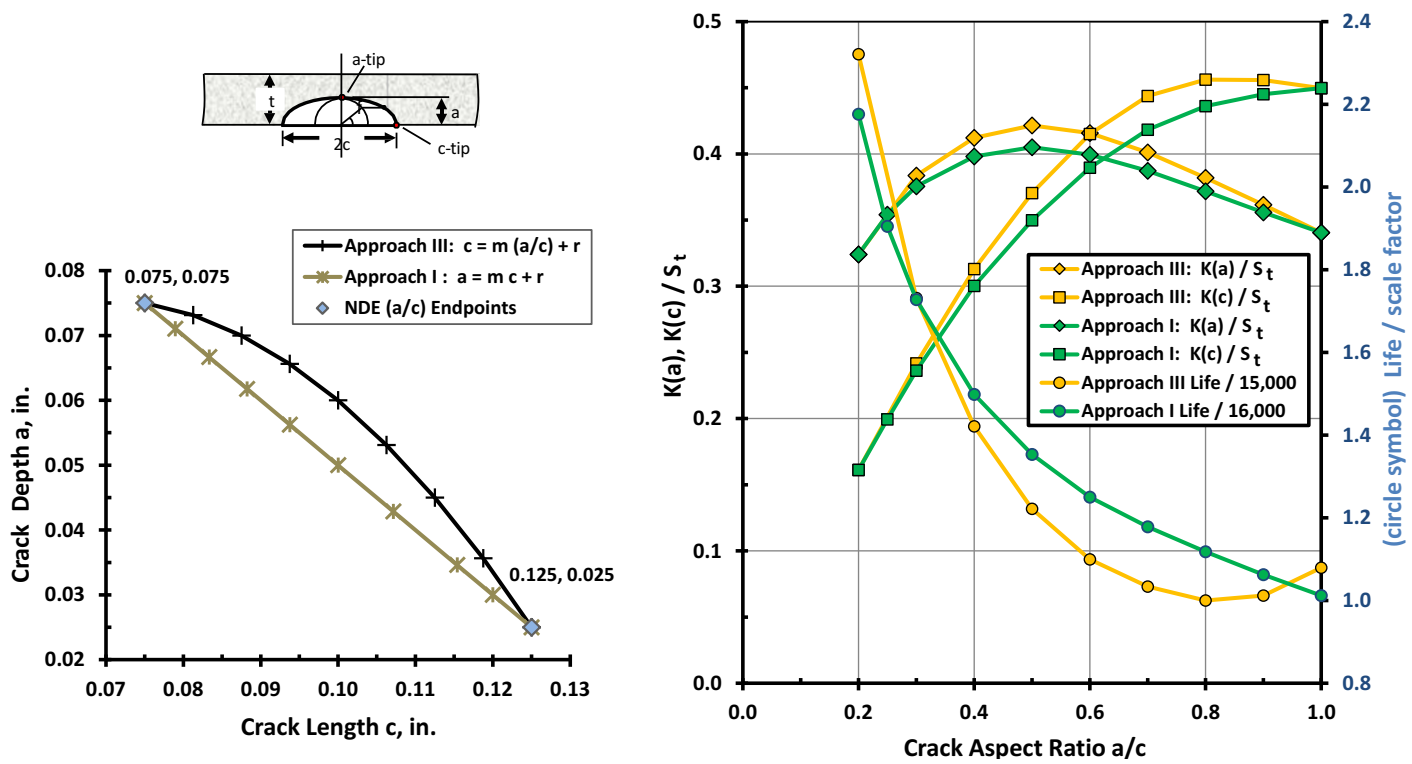

(a) Penetrant NDE aspect ratio endpoints and interpolated shapes using Approaches I and III.

(b) Initial crack tip $K(a), K(c)$ for interpolated crack shapes using Approaches I and III , and computed lives at each aspect ratio.

Figure 11. Penetrant NDE 5009 detectable crack aspect ratio endpoints, interpolated crack shapes, normalized initial stress intensity factors, and resulting lives at aspect ratios $(20 \mathrm{ksi}$ remote tension $\&$ plate thickness $=0.1$ in). 

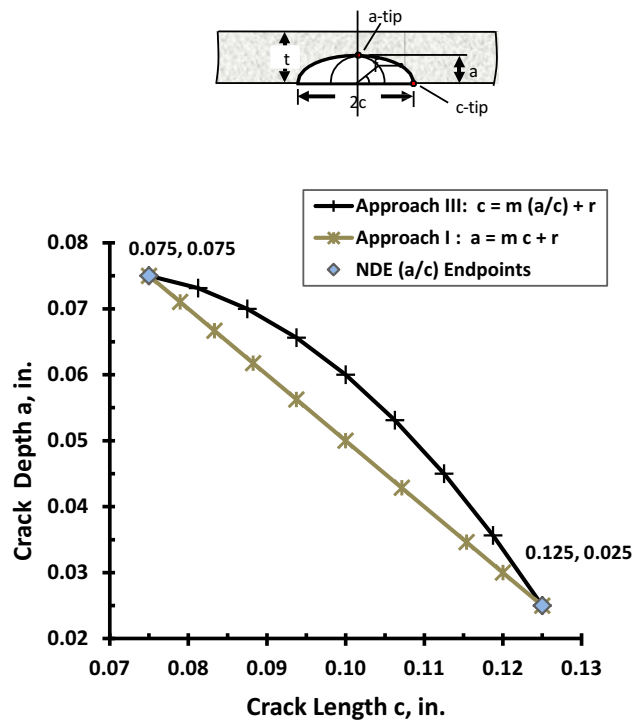

(a) Penetrant NDE aspect ratio endpoints and interpolated shapes using Approaches I and III.

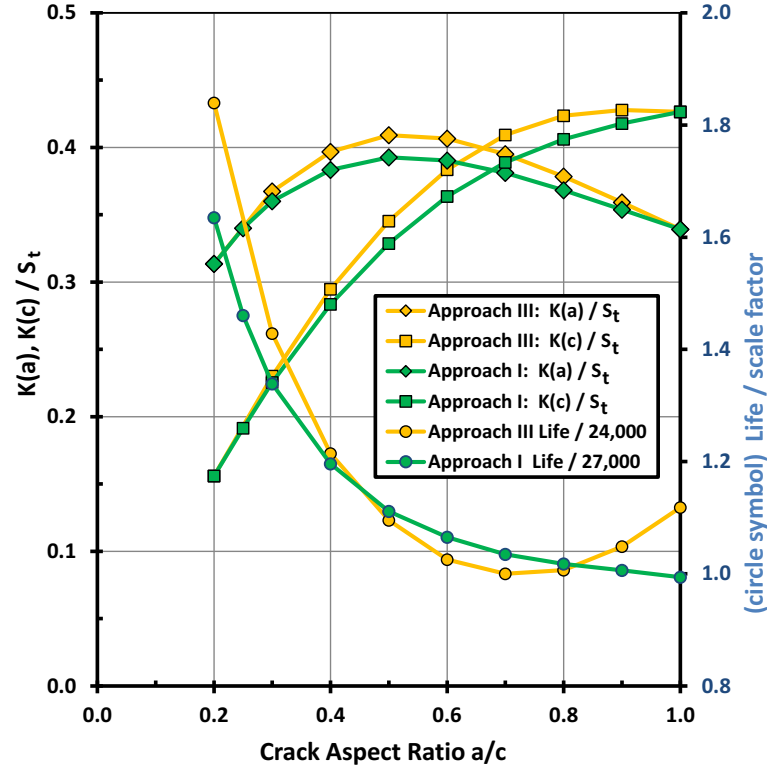

(b) Initial crack tip $\mathrm{K}(\mathrm{a}), \mathrm{K}(\mathrm{c})$ for interpolated crack shapes using Approaches I and III, and computed lives at each aspect ratio.

Figure 12. Penetrant NDE 5009 detectable crack aspect ratio endpoints, interpolated crack shapes, normalized initial stress intensity factors, and resulting lives at aspect ratios $(20 \mathrm{ksi}$ remote tension \& plate thickness $=0.12$ in).

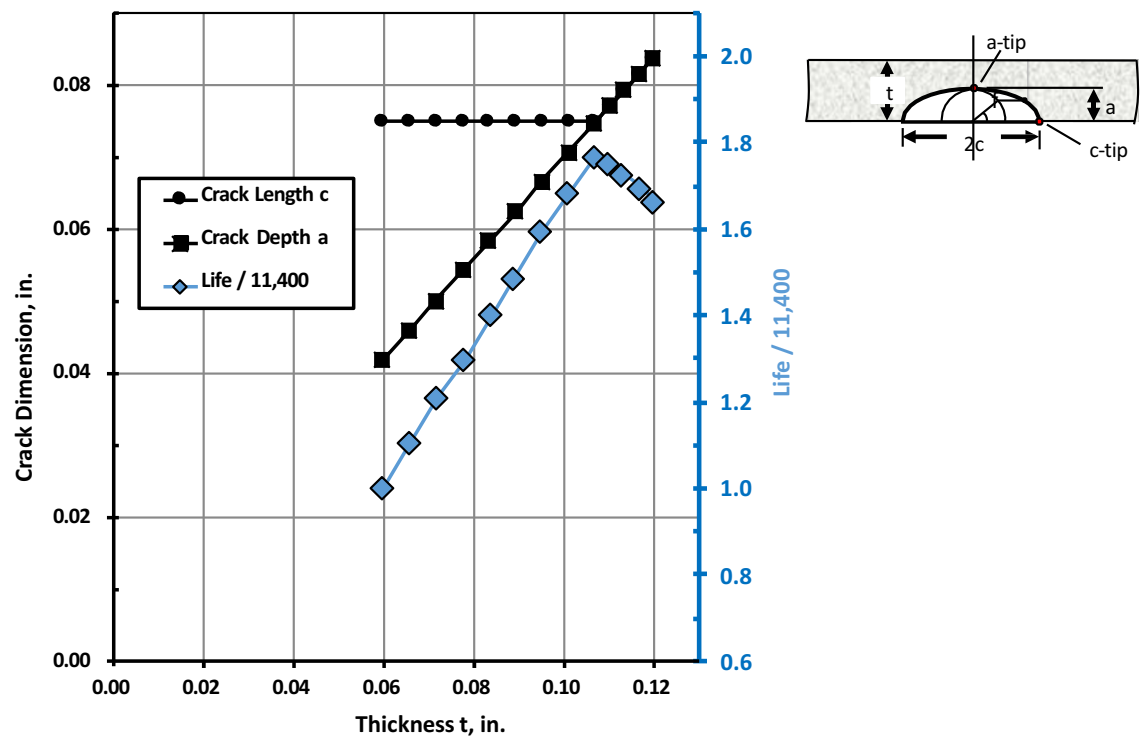

Figure 13. Radiographic NDE crack shapes and life calculations (20 ksi remote tension). 

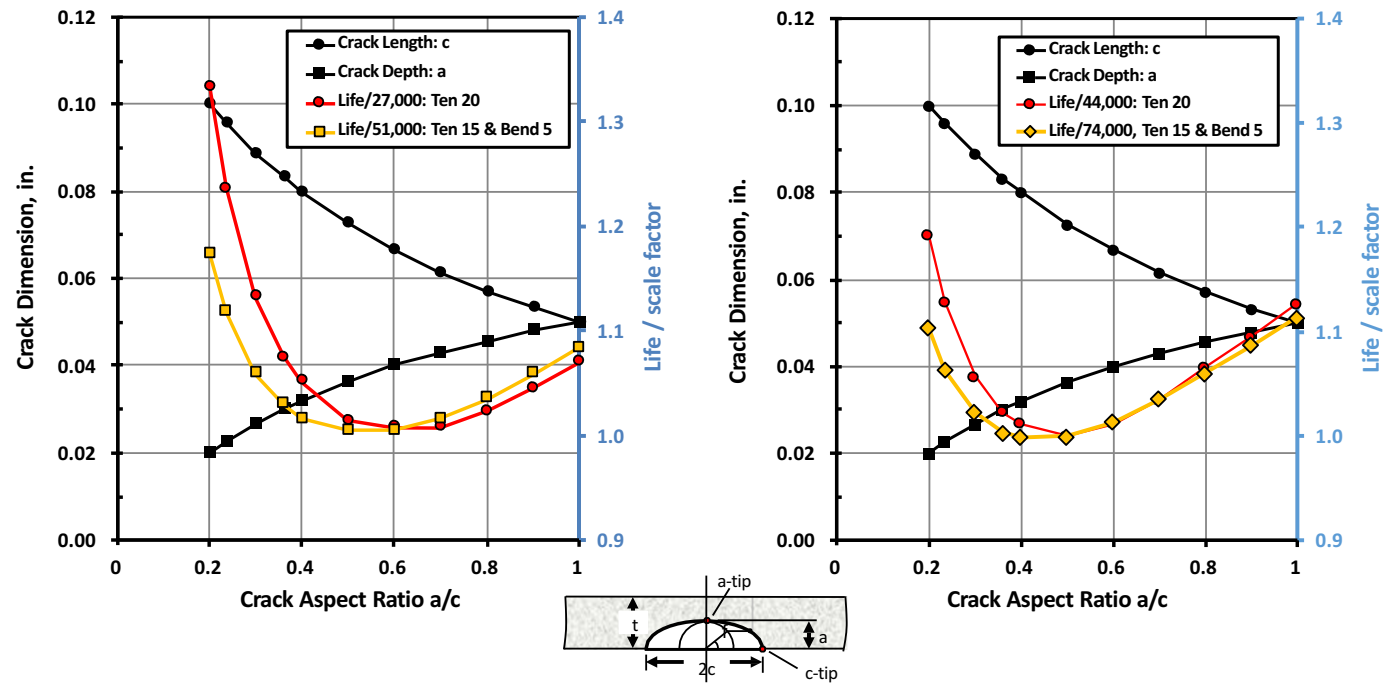

(a) Thickness of plate $=0.075$ in.

(b) Thickness of plate $=0.10$ in.

Figure 14. Comparison of lives for eddy current NDE crack shapes with remote tension (20 ksi) and tension (15ksi) + bending ( 5 ksi) loadings.
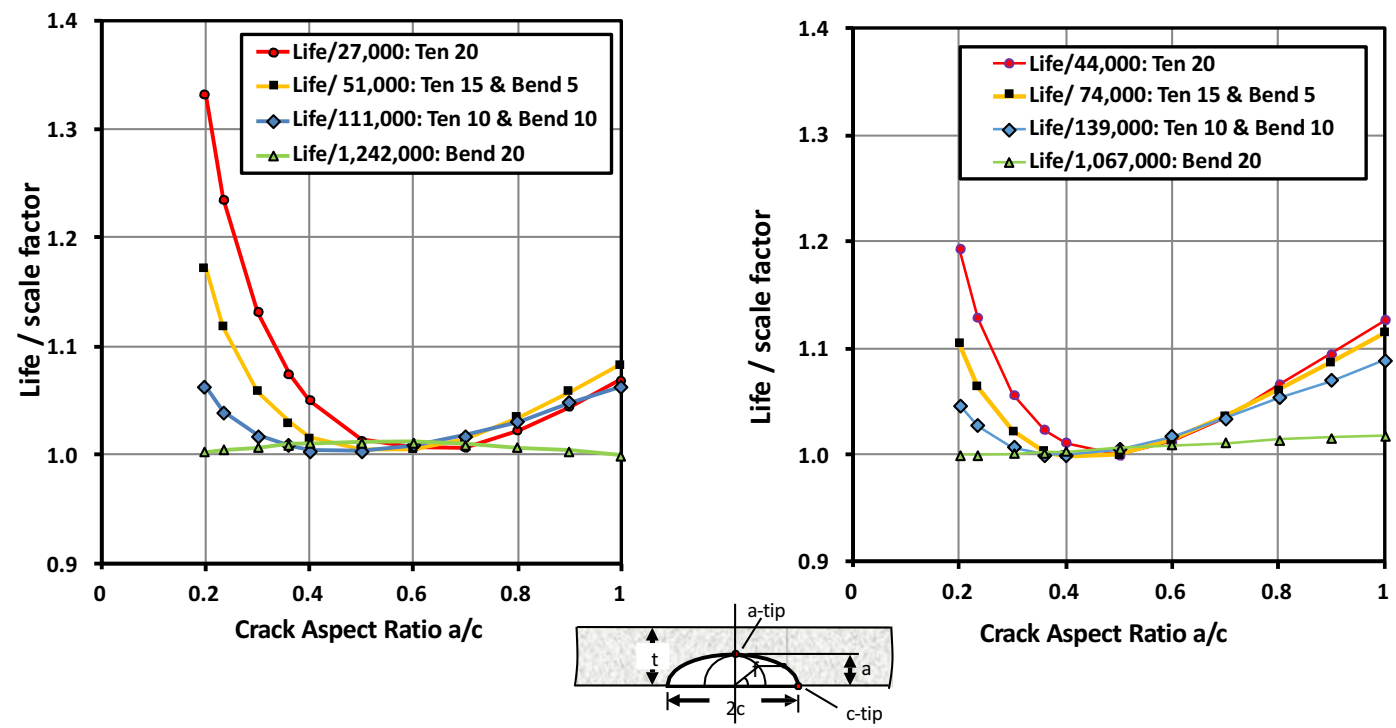

(a) Thickness of plate $=0.075$ in.

(b) Thickness of plate $=0.10$ in.

Figure 15. Comparison of lives for Eddy current NDE crack shapes remote tension (20 ksi) and various tension + bending loadings.

The assumption of linearity between $a$ and $c$ (Approach I) is more conservative than the approaches that assume that the area varies linearly between the two endpoints (Approaches IV \& V). Thus, Approaches IV and V are not recommended.

The assumption that the surface crack depth is a linear function of the aspect ratio between the two endpoints (Approach II) is less conservative than Approach I. Thus, Approach II is not recommended. Approach III has the advantage that it is more conservative than Approach I, so it is also evaluated with results shown in Figs. 11 and 12 for the 5009 PT NDE detectable crack aspect ratio endpoints.

The assumptions that the crack area is constant (Approaches VI and VII) has a logical inconsistency because the areas at the two endpoints are generally not the same. Thus, using the constant area of one of the NDE endpoints will have a discontinuity at the opposite endpoint. The approach that uses the smaller area (generally the smaller aspect ratio: Approach VII) will likely be unconservative relative to Approach I. The approach that uses the larger 
area (generally the larger aspect ratio: Approach VI) can be either conservative on nonconservative relative to Approach I. Further guidance from the NDE community is needed if Approach VI is preferable.

From this study, it is also noted that it is not clear how to predict the initial crack size for other aspect ratios if only one NDE detectable crack aspect ratio endpoint value (e.g., $(a / c)=1.0)$ is known. The use of an equal area assumption would appear to be inconsistent with the detectable crack aspect ratio endpoint sizes with different areas found in $5009^{4}$.

As noted previously, Approach III is more conservative than Approach I, and hence can be used as the method for interpolating between the $(a / c)$ ratio endpoints for all the reported results. However, the results reported for the ET and UT NDE methods were performed first using Approach I. Also, Approach I is appealing because it produces crack sizes that are on the straight line connecting the two endpoints, i.e., it produces $a, c$ points that are always closest to the known $a$, $c$, values at the $(a / c)$ ratio endpoints. Both Approach I and III are used to provide a comparison of their results for the PT NDE method evaluations described later.

Discussions with available NDE experts confirmed the 5009 NDE data values represent probability of detection data from historical controlled studies as cited in the standard and the data were not derived based on any a priori presumptions as to how crack shape $(a / c)$ or area or any other aspects affected the detectable crack sizes. In other words, at this time, it appears there is no information suggesting how to interpolate between the $(a / c)$ endpoints.

\section{Life Calculations}

Fatigue crack growth life predictions were performed assuming that LEFM is applicable. NASGRO ${ }^{\circledR}$ version 8.1 was used to perform the life predictions for a surface crack in a 3-inch-wide flat plate of 6061-T6 aluminum. The SC 30 stress intensity factor solution was selected for this configuration. In the first set of analyses, the plate was subjected to a remote tensile stress of $20 \mathrm{ksi}$ with a stress ratio $\left(\mathrm{R}=S_{\min } / S_{\max }\right.$ where $S$ is the remote applied stress) of 0.1. The lives of the crack that are shown in Figs. 8 through 15 are the lives for each of the interpolated initial $a$ and $c$ crack size aspect ratios for continuing cycles of the applied loadings up to the point when the crack transitions from a PTC (Part Through Crack) to a TC (Through Crack). Also, in these analyses, all easily available NASGRO ${ }^{\circledR}$ failure criteria checks (fracture toughness value $\mathrm{K}_{\mathrm{IC}}$, net section stress, plastic limit load, and Option $1 \mathrm{FAD}^{6}$ ) were used. All analyses predicted failure after the transition to a through crack (TC) for this material due to Option 1 FAD calculation; however, the life to the fracture event was not reported because once the PV leaks it has failed to perform its function.

Figures 8 through 12 present initial interpolated crack shapes and the resulting lives for the semi-elliptical cracks in a plate subjected to remote tensile stress of $S_{t}=20 \mathrm{ksi}$ (see Fig. 6). The four crack NDE methods presented in Table 1 are considered. Approach I that assumes a linear relationship between $a$ and $c$ of the two $(a / c)$ endpoints was used to determine intermediate $(a / c)$ crack sizes in all the analyses reported here. However, as noted previously, Approach III is more conservative, and results using both Approach III and Approach I appear in Figs. 11 and 12 that address PT NDE. Note that the Radiographic NDE is an exception that does not need an Approach method for interpolating sizes because the 5009 standard specifies that the crack sizes that vary are a function of plate thickness. These results are presented in Fig. 13.

For the other three NDE methods, that are all assessed using Approach I, a typical point $\mathrm{j}$ on the straight line connecting the $a$ and $c$ values of the endpoints is read off as $a_{j}$ and $c_{j}$. (Or alternatively, the linear equation for the line connecting the $a, c$ endpoints may be used to compute intermediate points.) The corresponding aspect ratio is computed as $\left(a_{j} / c_{j}\right)$. The combination of $a_{j}, c_{j}$, and $\left(a_{j} / c_{j}\right)$ initial crack sizes used in the analysis results are provided in plots of $a$ vs $c$, and their values are also plotted against the left hand side and abscissa of each plot showing crack life except Figs. 11 and 12. (Since both Approaches I and III are used for results reported in these two figures, it is not practical to show the crack sizes in the plots showing life.)

In the first procedure for determining crack $a$ and $c$ sizes for analysis as outlined above, round numbers for $(a / c)$, for example $(a / c)=0.3$ (as opposed to $(a / c)=0.3165$ ), are not easily obtained. If one requires round $(a / c)$ numbers, a second procedure may be followed. Simple algebra was used to transform the linear relationship of $a$ and $c$ (Eq. 1) into relationships between $c$ and $a / c$ (Eq. 4) and $a$ and $a / c$ (Eq. 5). The $a$ vs $c$ fit can be expressed as

$$
\mathrm{a}=\mathrm{mc}+\mathrm{r}
$$

\footnotetext{
${ }^{6} \mathrm{FAD}=$ failure assessment diagram
} 
the slope $m$ and the intercept $r$ can be evaluated using the two end points of $(a / c)$ values of 0.2 and 1 , as

$$
\mathrm{m}=\frac{a_{0.2}-a_{1.0}}{c_{0.2}-c_{1.0}} ; \quad r=a_{0.2}-m * c_{0.2}
$$

Dividing both sides of the Eq. (1) by $c$ gives

$$
(a / c)=m+\frac{r}{c}
$$

Rewriting Eq. (3)

$$
c=r /[(a / c)-m]
$$

then, once $c$ is known for the selected $(a / c), a$ is easily obtained using

$$
a=(a / c)^{*} c
$$

For any given value of $(a / c)_{k}$, (for example $a / c=0.3$ ), the values of $c_{k}$ can be calculated using Eq. (4) with the slope $m$ and the intercept $r$ given by Eq. (2). The corresponding value of $a_{k}$ can be calculated using Eq. (5). The values of $c_{k}$ and $a_{k}$ and $(a / c)_{k}$ obtained by this procedure plots on the same straight line between the two end points as obtained by the first procedure. Example plots of the interpolated $a, c$ values are provided with the estimated detectible crack sizes. The crack initial sizes are inputs for the analysis results shown in Figs. 8 through 12.

The life of the cracked component was calculated using NASGRO ${ }^{\circledR} 8.1$, as mentioned above, for each selected $\left(a_{j} / c_{j}\right)$ combination. The life curve is plotted using the right hand ordinate in each figure. This crack life is normalized by a convenient number that is approximately the minimum life computed for each case. The normalizing numbers or "scale factors" cause the minimum life to plot at 1.0 on the plot right size ordinate, but actual life is the "scale factor" times the ordinate.

Figures 8 and 9 for two plate thicknesses show that the minimum life for the initial crack size determined by eddy current NDE does NOT occur at the $(a / c)$ range endpoints, but somewhere near the aspect ratio of about 0.6 for a 0.075 -inch-thick plate and of about 0.5 for a 0.1 -inch-thick plate. Figure 10 shows that minimum life for ultrasonic NDE initial cracks does NOT occur at the endpoints, but near $(a / c)$ of about 0.4 for the 0.10 -inch-thick plate.

Figure 11(b) illustrates the effects of using Approach III versus Approach I to interpolate crack sizes for aspect ratios between the 5009 crack aspect ratio endpoints for penetrant NDE. When Approach I is used, the minimum life occurs at the $(a / c)=1.0$ endpoint. When Approach III is used, the minimum life occurs at an $(a / c)$ value of 0.8 . There are two factors in play causing this effect. One factor is the influence of the surface crack $(a / c)$ ratio on stress intensity factor (SIF), as shown in Fig. 5. The other factor results from the larger crack $a$ and $c$ dimensions that develop from the Approach III interpolated $(a / c)$ ratio endpoints as compared to those using Approach I. The two interpolated crack shape curves, shown in Fig. 11(a), demonstrate the larger crack sizes that result from the use of Approach III. Whether these interpolated shapes are realistic or artificial cannot be determined without addition NDE data on crack sizes with equal probability of detection at intermediate $(a / c)$ aspect ratios. The integrated effects of the $(a / c)$ effects on the SIF, and the differences in crack sizes at each interpolated aspect ratio are reflected by the curves showing the SIF, i.e., $\mathrm{K}(a)$ and $\mathrm{K}(c)$ for both Approach I and III in Fig. 11(b). The SIFs resulting from Approach III are larger in the middle of the crack aspect ratio range, and these tend to move the location where the life is a minimum towards the center of the crack aspect ratio range. There is also a smaller effect due to plate thickness as illustrated by comparing the results shown in Fig. 12 for a 0.12-inch-thick plate with those in Fig. 11 for a 0.1 -inch-thick plate.

In summary, the different aspect ratios with the minimum life shown in Figs. 8 to 12 are due to combined effects of both the different values for each NDE method of detectable initial crack sizes, $a$, $c$, at the $(a / c)$ ratio endpoints as specified in 5009 and the method of interpolating intermediate crack sizes between these a/c ratio endpoints. The effect of plate thickness differences on the crack aspect ratio with minimum life is small for the analyses reported here. However, larger variations of thickness, and much smaller plate thicknesses, may occur in PV, especially in flight COPV liners. Plate thicknesses and variations of thickness in those applications may have a much larger effect on life and aspect ratio effects. However, those situations are outside the scope of this study.

Figure 13 presents the crack shape and life calculations for radiographic NDE PTC cracks. Here the crack depth, $a$, is a function of the thickness of the plate. Hence crack initial lengths $a$ and $c$ are plotted against the thickness of 
the plate in this figure. For $t<0.107$ in., surface crack half-length is a constant $c=0.075$ in. The crack depth, $a$, on the other hand is a linear function of the thickness $(0.7 t)$. For $t>0.107$ in., the surface crack half-length $c$, is also a linear function of thickness $(0.7 t)$. This combination of crack shapes results in a normalized life that was nearly linear up to $t=0.107 \mathrm{in}$. and decreased sharply for thicker plates. Therefore, the use of estimated minimum detectible crack sizes for RT will require a careful evaluation of the influence of plate thickness.

\section{$X$. Tension and Bending Loadings}

Computations for combinations of remote tensile and bending loadings for the eddy current (ET) NDE method initial detectable cracks are described next. For each of these loading conditions, the same eddy current initial PTC sizes using Approach I for interpolation of intermediate crack sizes shown previously in Figs. 8 and 9 are used. These PTC sizes appear again in Fig. 14. Four different remote loadings are analyzed including: (A) $S_{t}=20 \mathrm{ksi}$ tensile loading, (B) $\mathrm{S}_{\mathrm{t}}=15 \mathrm{ksi}$ tensile plus $\mathrm{S}_{\mathrm{b}}=5 \mathrm{ksi}$ bending loadings, (C) $\mathrm{S}_{\mathrm{t}}=10 \mathrm{ksi}$ tensile plus $\mathrm{S}_{\mathrm{b}}=10 \mathrm{ksi}$ bending loadings, and (D) $S_{t}=0$ tensile with $S_{b}=20$ ksi bending loading. These stress loadings are shown in Fig. 6 (b) as $S_{t}$ and $S_{b}$. Note that the four cases were selected to have the same maximum stress acting on the outer surface and the crack $c$-tip. These analyses demonstrate the tension loading has a much shorter life than cases consisting partly of bending stress.

Figure 14 presents comparison of normalized lives for the first two cases (A) and (B) for plate thicknesses of $0.075 \mathrm{in}$. and $0.10 \mathrm{in}$. As expected, the pure tensile loading has a lower life for most aspect ratio crack configurations for both plate thicknesses considered. The minimum life for the (B) loading occurs at a slightly smaller aspect ratio for the two plate thicknesses as compared to the (A) loading minimum locations -- that were previously shown in Figs. 8 and 9 and noted to be about 0.6 for the 0.075 -inch-thick plate and about 0.5 for the 0.1 -inch-thick plate. The change from all tension to tension plus a small bending loading shown in Fig. 14 has not produced a significant shift in the crack aspect ratio where the minimum life occurs. (Note the "scale factor" causes the Tension only $20 \mathrm{ksi}$ loading (labeled "Ten 20") to appear to have a larger ordinate (life) than the loading with 15 ksi tension and 5 ksi bending (labeled "Ten 15 \& Bend 5."). In actuality, the $20 \mathrm{ksi}$ tension result has a much smaller life when the plot life ordinate is correctly interpreted using the "scale factors" shown in the plot legend - these factors are the minimum life for each analysis.)

Figure 15 presents lives as a function of aspect ratio of the cracks for the same eddy current initial PTC sizes, for the four loading cases listed above. As expected, the lives of all the cracks becomes larger as the tension component decreases and the bending component increases, even though the total stress at the crack $c$-tip is always the same. The smallest value of life appears to occur at an $(a / c)$ value of about 0.6 for the 0.075 -inch-thick plate and 0.4 to 0.5 for the 0.10 -inch-thick plate. The crack aspect ratio where the minimum life occurs has not changed significantly with the increasing bending loading cases studied here. However, for loading cases where the bending is fully reversed, or where complex stress gradients occur, it is expected the $(a / c)$ value where minimum life occurs could be strongly affected.

\section{Other Considerations}

The standards S 080-1998 and S 081-2006 have different requirements when safe-life (of 4 service life times) and leak before burst (LBB) needs to be demonstrated. In these calculations, the applicable fracture toughness (e.g., $\mathrm{K}_{\mathrm{IC}}, \mathrm{K}_{1 \mathrm{C}}, \mathrm{K}_{1 \mathrm{e}}, \mathrm{K}_{\mathrm{C}}$ ) of the material should be considered. The definitions of various toughness values are given in NASA-STD-5019A 9 and presented in the box below. The values of the toughness used in the analyses need to be justified and shown to be valid for the materials and thicknesses used in the vessels and the crack configuration being evaluated.

The manufacturing and fabrication of the vessels generally leaves residual stresses that may influence the behavior of cracks. These residual stresses have complex through-the-thickness distributions and may vary from location to location on the PV. Often, the presences of residual stresses are suspected, but the magnitudes and

distributions are unknown. Methods that bound the influence of residual stresses on DT may determine if quantitative characterization of residual stresses is necessary. 


\section{Various Toughnesses Used in the DT Analyses}

$\mathrm{K}_{\mathrm{c}}$ : Plane stress fracture toughness.

The value of stress intensity factor $\mathrm{K}$ at the tangency between a crack extension resistance curve (R-curve) and the configuration-dependent applied K curve (ASTM E1823, Standard Terminology Relating to Fatigue and Fracture Testing). This crack extension occurs under conditions that do not approach crack-tip plane strain. The R-curve and $\mathrm{K}_{\mathrm{c}}$ vary with the material, specimen size, and thickness. Kc is used in NASGRO ${ }^{\circledR}$ to represent fracture toughness as a function of thickness for use in crack growth calculations.

$\mathrm{K}_{\mathrm{Ic}}$ : Plane strain fracture toughness.

The crack extension resistance under conditions of crack-tip plane strain in Mode I for slow rates of loading under predominantly linear-elastic conditions and negligible plastic-zone adjustment that is measured by satisfying a standardized procedure with validity requirements (ASTM E399, Standard Test Method for Linear-Elastic Plane-Strain Fracture Toughness $\mathrm{K}_{\mathrm{Ic}}$ of Metallic Materials). Another quantity, $\mathrm{K}_{\mathrm{II}}$, defined for conditions with limited plasticity from $\mathrm{J}_{\mathrm{Ic}}$ may also be useful (ASTM E1820).

$\mathrm{K}_{\mathrm{Ie}}$ : Effective fracture toughness for a surface or elliptically shaped crack.

The toughness is based on residual strength and the original crack dimensions. This parameter is meaningful only when crack-tip plastic zones are small and stable crack growth before failure is generally absent (ASTM E740/E740M, Standard Practice for Fracture Testing with Surface- Crack Tension Specimens, main body and section X1.2). For conditions with plastic effects and welldefined crack-tip stress fields with fracture controlled by crack initiation, an approach involving constraint may be applicable (ASTM E2899). Testing of flaws in specimens representative of the structure is needed to determine damage tolerance for plasticity conditions when crack-tip stress fields collapse. $\mathrm{K}_{\mathrm{Ie}}$ is used in NASGRO ${ }^{\mathrm{B}}$ for analyses of crack growth.

$\mathrm{K}_{\mathrm{EAC}}$ :

The largest value of the stress intensity factor at which crack growth is not observed for a precracked through-crack specimen of specified material, environment, and thickness that is tested for a significant duration in accordance with ASTM E1681.

$\mathrm{K}_{\mathrm{IEAC}}$ :

The largest value of the stress intensity factor at which crack growth is not observed for a precracked through-crack specimen of specified material, environment, and thickness that is sufficient to meet requirements for plane strain and is tested for a significant duration in accordance with ASTM E1681.

$\mathrm{K}_{\text {Iscc: }}$ :

$\mathrm{K}_{\mathrm{EAC}}$ is often denoted as $\mathrm{K}_{\mathrm{Iscc}}$ in the literature.

\section{Recommendations}

The results presented in this paper illustrate how the interpretation of the implementation details of the AIAA S080 and S081 standards can influence the DT life predictions of COPVs. The requirement that safe-life be demonstrated for the minimum detectible flaw sizes for specific NDE methods (as assessed by estimated 90/95 POD) requires an interpretation of the initial part through crack size between the aspect ratio values (endpoints) listed in the NASA STD 5009 (generally at an aspect ratio of $a / c=0.2$ and 1). The choice of interpolation method for the part through crack sizes between the listed NDE endpoints can influence the analytically predicted life. The following recommendations are made based on the findings of this paper:

- Table 1 of NASA STD 5009 that lists the minimum detectible flaw sizes of various NDE techniques should be updated to include a minimum of three aspect ratios for each technique. It is recognized that this is a significant undertaking, but it is necessary to eliminate the ambiguity present in the current situation.

- In the interim, i.e. before Table 1 of NASA STD 5009 can be updated, the community should adopt a standard approach for the interpolation of part through crack sizes from the listed NDE endpoints. There are two possible choices: Approach I or III as discussed in the paper. Without any other information, 
Approach III, as the most conservative, is a logical choice to be the standard method for interpolation between the listed crack length $(c)$ and depth $(a)$ values.

\section{Concluding Remarks}

Some observations on damage tolerance (DT) analyses of pressure vessels (PVs) and composite overwrap pressure vessels (COPVs) are discussed. The two standards that are applicable are AIAA S 080 for metallic PVs and S 081 for COPVs.

As is customary in any DT analyses, a flaw that is the estimated minimum reliably detectible crack is assumed to exist in the pressure vessel and the crack is oriented perpendicular to the maximum tensile principal stress. The DT analysis then proceeds to demonstrate safe-life (four lifetimes of the component) to satisfy the standard requirements. These analyses should use available failure criteria to ensure the results are the minimum life. The minimum detectible crack sizes are given in the NASA-STD-5009 for various NDE methods. In this paper, eddy current, penetrant, radiographic, and ultrasonic methods were considered. NASA-STD-5009 specifies a semi-elliptic surface crack with aspect ratios $(a / c)-a$ is the crack depth and $c$ is the semi-surface length - of 0.2 and 1 for the eddy current, penetrant, and ultrasonic NDE methods. No intermediate $(a / c)$ NDE crack size data are provided in the standard. Crack size is specified in this standard as a function of thickness for the radiographic method

While the AIAA standards appear to be clear on the procedures/processes needed for demonstrating safe-life, various questions arise from these requirements. For example, how is the requirement 'in the range of $(a / c)=1.0$ and $0.2^{\prime}$ ' interpreted? How many crack aspect ratios $(a / c)$ need to be considered? Does the stress-intensity factor need to be examined at every point on the crack front? Where is the stress-intensity factor maximum along the crack front? Are the conclusions drawn for a particular liner thickness valid for others? In this paper, some of these requirements are highlighted and some clarifications are offered.

The maximum stress-intensity factor location occurs only at the $a$-tip or the $c$-tip and not elsewhere along the crack front. Several approaches were examined for initial crack sizes between the $a / c$ ratio endpoints as specified for the eddy current, penetrant, and ultrasonic NDE methods. The approaches included: linear relationships between $a$ and $c$ (Approach I); $a$ and $(a / c) ; c$ and $(a / c)$ (Approach III); $a$ and the area of the crack; $c$ and the area of the crack; constant area determined by $(a / c)=1$; and constant area determined by $(a / c)=0.2$. Approach I that assumes a linear relationship between $a$ and $c$ at the two endpoints is a simple, historical method, but Approach III is more conservative. If no other detectable crack size information is available to guide the interpolation, Approach III is the logical choice at this time. However, the ambiguity that results from only the two existing $(a / c)$ endpoints could be eliminated if a third $(a / c)$ point is established for each NDE method. Also, in that event, additional interpolation methods may be considered.

Using the cited approaches, interpolated crack shapes were constructed between the 5009 aspect ratio endpoints for the eddy current, penetrant, and ultrasonic NDE methods and the lives of each of these crack configurations in a plate subjected to remote tensile loading was evaluated using NASGRO ${ }^{\circledR} 8.1$ software. These calculations using Approach I demonstrate that the eddy current and ultrasonic NDE methods 5009 detectable crack sizes cause the smallest life to not occur at the two $(a / c)$ endpoints, but at an intermediate configuration in the range $(a / c)$ of 0.4 to 0.6. The penetrant NDE method has smallest life at $(a / c)$ of 1.0 when crack size aspect ratio is interpolated using Approach I. However, the result shifts to an intermediate $(a / c)$ of 0.7 to 0.8 when crack size aspect ratio is interpolated using Approach III. The effect of plate thickness differences on the crack aspect ratio with minimum life is small for the analyses reported here. However, larger variations of thickness, and much smaller plate thicknesses, may occur in PV, especially in flight COPV liners. In general, the crack size aspect ratio where minimum life occurs is expected to depend on the detectable crack size aspect ratio endpoints, the interpolation method, and the loading conditions. As such, analyses that interrogate only the two endpoints of $(a / c)=0.2$ and 1 likely result in unconservative life predictions. The standard practice needs to be reviewed based on the results in this paper.

\section{References}

${ }^{1}$ Ecord, G. M., “Apollo Experience Report-Pressure Vessels," NASA TN-D 6975, September 1972. [This reference summarized the experiences with PVs prior and up to the Apollo program.]

${ }^{2}$ ANSI/AIAA S-080-1988, Standard for Space Systems-Metallic Pressure Vessels, Pressurized Structure, and Pressure Components, September 13, 1999.

${ }^{3}$ ANSI/AIAA S-081A-2006, Standard for Space Systems - Composite Overwrapped Pressure Vessels, July 24, 2006.

${ }^{4}$ NASA Standard, Nondestructive Evaluation Requirements For Fracture-Critical Metallic Components, NASA-STD-5009, April 2008. 
${ }^{5}$ NASGRO $^{\circledR}$, Fracture Mechanics and Fatigue Crack Growth Analysis Software, version 8.1, May, 2016. Developed under a Space Act Agreement between NASA Johnson space Center and Southwest Research Institute ${ }^{8}$; www.nasgro.com.

${ }^{6}$ Raju, I. S., and Newman, J. C., Jr., "Stress-Intensity Factors for a Wide Range of Semi-Elliptical Surface Cracks in FiniteThickness plates," Engineering Fracture Mechanics, Vol. 11, 1979, pp. 817-829.

${ }^{7}$ Newman, J. C., Jr. and Raju, I. S., “An Empirical Stress- Intensity Factor Equation for the Surface Crack," Engineering Fracture Mechanics, Vol. 15, No. 1-2, 1981, pp. 185-192.

${ }^{8}$ Murakami, Y. (Ed.), Stress Intensity Factors Handbook, Volumes 1 and 2, Pergamon Books, Ltd, 1987.

${ }^{9}$ NASA Standard, Fracture Control Requirements For Spaceflight Hardware, NASA-STD-5019A, February 2016.

${ }^{10}$ King, J. R., Julian, P., and Johnson, K. R., "Space Shuttle Orbiter Fracture Control Plan," Space Division Rockwell International, SD73-SH-0082A, September 1974. 